\title{
MAGNETIC GUIDING OF MASS FLOW IN ERUPTIONS \\ OF CATACLYSMIC VARIABLES
}

\author{
F. Meyer \\ Max-Planck-Inst. f. Phys. \& Astrophys., München
}

It is suggested that the superhump phenomenon in eruptions of cataclysmic variables like VW Hy 1 indicates a continuous white light flaring in magnetic flux tubes connecting a magnetic spot on the convective dM secondary and the disk whose Keplerian rotation provides the driving energy by twisting up the magnetic field. Due to the gravitational (and centrifuga1) potential difference between the surface of the secondary and the "deeper" disk material a continuous evaporation-condensation mass flow from the secondary to the disk must occur. This can account for the 100-fold increase in mass flow in the disk despite the fact that the ordinary Roche lobe overflow mass flow is not affected, as indicated by the unvaried brightness of the regular accretion spot at the disk. This model interprets the supermaxima eruptions as a consequence of a magnetic cycle on the secondary that periodically immerses the accretion disk in strong magnetic fields. Due to the strong dependence of dipolar field strength on distance from the secondary surface this might explain why the superhump phenomenon is mainly found in the closer dwarf novae. Required field strengths and observed spot sizes are in good agreement with expectations and observations from smaller dM stars like BY Draconis-type stars and flare stars. 